\title{
IS26-Mediated Genetic Rearrangements in Salmonella Genomic Island 1 of Proteus mirabilis
}

\author{
Xue-Chun Wang ${ }^{\dagger}$, Chang-Wei Leit, Zhuang-Zhuang Kang, Yu Zhang and \\ Hong-Ning Wang* \\ Animal Disease Prevention and Food Safety Key Laboratory of Sichuan Province, Key Laboratory of Bio-Resource \\ and Eco-Environment of Ministry of Education, College of Life Sciences, Sichuan University, Chengdu, China
}

Salmonella genomic island 1 (SGl1) is an integrative mobilizable element integrated into the chromosome of bacteria, which plays an important role in the dissemination of antimicrobial resistance genes. Lots of SGl1 variants are found mainly in Salmonella enterica and Proteus mirabilis. In this study, a total of 157 S. enterica and 132 P. mirabilis strains were collected from food-producing animals in Sichuan Province

OPEN ACCESS

Edited by:

Monika Dolejska

University of Veterinary and Pharmaceutical Sciences Brno,

Czechia

Reviewed by: Xiaodong Xia,

Northwest A\&F University, China Jianmin Zhang, South China Agricultural University,

China

*Correspondence:

Hong-Ning Wang

whongning@163.com

${ }^{\dagger}$ These authors have contributed equally to this work

Specialty section: This article was submitted to Antimicrobials, Resistance and Chemotherapy,

a section of the journal

Frontiers in Microbiology

Received: 18 May 2019 Accepted: 13 September 2019 Published: 24 September 2019

Citation:

Wang X-C, Lei C-W, Kang Z-Z,

Zhang $Y$ and Wang H-N (2019)

IS26-Mediated Genetic

Rearrangements in Salmonella

Genomic Island 1 of Proteus mirabilis.

Front. Microbiol. 10:2245.

doi: 10.3389/fmicb.2019.02245 of China between December 2016 and November 2017. Detection of the SGl1 integrase gene showed that three $S$. enterica and five $P$. mirabilis strains were positive for SGl1, which displayed different multidrug resistance profiles. Five different SGl1 variants, including two novel variants (SGl1-PmBC1123 and SGl1-PmSC1111), were characterized by whole genome sequencing and PCR linkage. In two novel SGl1 variants, IS26-mediated rearrangements resulted in large sequence inversions of the MDR regions extending outside the SGl1 backbone. The sul3-type III class 1 integron (5'CS-sat-psp-aadA2-cm/A1-aadA1-qacH-IS440-sul3) and gene cassettes aac(6')Ib-cr-blaOXA-1-catB3-arr-3 are found in SGl1-PmSC1111. Mobilization experiments indicated that three known variants were conjugally mobilized in trans to Escherichia coli with the help of a conjugative IncC plasmid. However, the two novel variants seemed to lose the mobilization, which might result from the sequence inversion of partial SGI1 backbone. The identification of the two novel SGI1 variants in this study suggested that IS26-mediated rearrangements promote the diversity of SGI1.

Keywords: Salmonella, Proteus, genomic island, SGI1, multidrug resistance, IS26

\section{INTRODUCTION}

Genomic islands (GIs), such as integrative and conjugative elements (ICEs) and integrative mobilizable elements (IMEs), are distinct regions integrated into the chromosome of bacteria and acquired via horizontal transfer (Bellanger et al., 2014; Partridge et al., 2018). GIs often contain various genes endowing theirs hosts with new traits, like antimicrobial resistance and virulence that enhance bacterial adaptation to environment (Bellanger et al., 2014). Salmonella genomic island 1 (SGI1) is an IME initially identified in the multidrug resistance (MDR) Salmonella Typhimurium DT104 clone (Boyd et al., 2001). SGI1 (42.4 kb) is comprised of a backbone containing 28 ORFs (S001-S027 and S044) and a $13 \mathrm{~kb}$ MDR region that consists of a complex In4-type class 1 integron named In104 (Boyd et al., 2001; Levings et al., 2005). It can form extrachromosomal circular form and is specifically mobilized in trans by conjugative IncA/C plasmids (Doublet et al., 2005; Douard et al., 2010). In recent years, the mobilization mechanism of SGI1 with the help of IncA/C 
plasmids has been revealed in some studies and many aspects have been explored including the basic transfer elements (Carraro et al., 2014; Kiss et al., 2015, 2019; Siebor et al., 2016). SGI1 was reported in Proteus mirabilis in 2007 (Ahmed et al., 2007), and recently found in Morganella morganii, Providencia stuartii and Escherichia coli (Schultz et al., 2017a; Cummins et al., 2019; Soliman et al., 2019), indicating that SGI1 has a broad host bacterial range and has the potential to spread among enterobacteria.

Since the first report of SGI1 in S. Typhimurium DT104, many different SGI1 variants have been described (Hall, 2010; Bi et al., 2011; Siebor and Neuwirth, 2011, 2013; Lei et al., 2014; Lei et al., 2015; Qin et al., 2015; Schultz et al., 2015; Bie et al., 2018; de Curraize et al., 2018), most of which result from various insertion sequences, homologous recombinations, transpositions, and loss or exchange of gene cassettes within the MDR region (Hall, 2010). A deletion of 2,780 bp in size from part of ORFs S005 to S009 that is replaced by ISVch4 is found in some variants like SGI1-K (Doublet et al., 2008). Besides, several SGI1 related islands (SGI1, PGI1, AGI1, PGI2 and GIPmi1) have been reported (Levings et al., 2008; Siebor and Neuwirth, 2014; Girlich et al., 2015; Hamidian et al., 2015; Lei et al., 2018b; Siebor et al., 2018, 2019), all of which incorporate into chromosomes at a specific location ( $3^{\prime}$ end of the trmE gene) as SGI1. SGI1 and related islands often harbor various antimicrobial resistance genes including carbapenems resistance gene bla $a_{\mathrm{NDM}-1}$ (Girlich et al., 2015), extended-spectrum $\beta$-lactamase (ESBL) genes bla $a_{\mathrm{VEB}-6}$ and bla $a_{\text {CTX-M-15 }}$ (Siebor and Neuwirth, 2011; de Curraize et al., 2018), and fluoroquinolones resistance genes qnrA1 and qnrB2 (Siebor and Neuwirth, 2011; Lei et al., 2014), indicating SGI1 and related islands are important vehicles for clinically important resistance genes. Recently, many variants of SGI1 and SGI1related islands have been characterized in $P$. mirabilis, which are summarized in Supplementary Table S1. These variants were found in P. mirabilis isolates from food, human, poultry, swine and other animals such as dog and horse.

In the present study, we characterized the SGI/PGI genomic islands in Salmonella enterica and P. mirabilis of food-producing animal origin in Sichuan Province of China and described two novel SGI1 variants in $P$. mirabilis.

\section{MATERIALS AND METHODS}

\section{Bacterial Strains and Detection of SGI1 and Relative Islands}

A total of 157 S. enterica strains (61 from swine and 96 from chicken) and $132 \mathrm{P}$. mirabilis strains (74 from swine and 58 from chicken) isolated from diseased tissues or anal swabs of animals among 30 poultry and 30 swine farms in Sichuan Province between December 2016 and November 2017. All isolates were identified using an automated system (BD Diagnostic Systems, Sparks, MD, United States). The presence of SGI/PGI/AGI/GIPmil was screened by PCR targeting the integrase gene (the primers used to detection are listed in Supplementary Table S2) (Schultz et al., 2017b). Positive PCR products were sent to Chengdu Qingke Biological Engineering
Technology \& Services Co., Ltd., and sequenced by ABI 3730xl DNA Sequencer (Applied Biosystems, United States).

\section{Antimicrobial Susceptibility Testing}

Antimicrobial susceptibility testing for strains positive for SGI1/PGI1 was determined by the disk diffusion method according to CLSI guidelines. Antimicrobial agents included ampicillin (AMP), amoxicillin-clavulanic acid (AMC), cefoxitin (FOX), cefotaxime (CTX), ceftriaxone (CRO), chloramphenicol (CHL), florfenicol (FFC), nalidixic acid (NAL), ciprofloxacin (CIP), streptomycin (STR), spectinomycin (SPT), apramycin (APR), doxycycline (DOX), trimethoprim (TMP), sulfizoxazole (SUL), trimethoprim-sulfamethoxazole (SXT) and polymyxin B (PB). E. coli ATCC25922 was used as a quality control strain.

\section{Whole Genome Sequencing and Analysis}

All SGI/PGI-positive strains were sequenced using Illumina HiSeq platform (400-bp paired-end reads with about 200 -fold average coverage). The draft genomes were assembled using software SPAdes_3.12.0. The gaps among contigs that carried SGI1 fragments were filled in by PCR linkage. Because the complete genetic structures of SGI1 in strains PmBC1123 and PmSC1111 could not be assembled by PCR linkage, whole genomes of those two strains were further sequenced using PacBio RS II sequencing instrument (100-fold average read depth). The chromosomes were assembled into one scaffold using software SMRT portal v.3.2.0. The MDR regions were confirmed by PCR linkage between regions belonging to non-repeated genetic elements. Multi-locus sequence type of S. enterica and acquired antimicrobial resistance genes were identified by MLST $2.0^{1}$ and ResFinder $3.1^{2}$, respectively. The complete nucleotide sequences of SGI1 variants were analyzed using the BLAST programs ${ }^{3}$. SNPs from genomes of the strains positive for SGI/PGI were called and a phylogeny based on the concatenated alignment of the high quality SNPs was inferred using CSI Phylogeny $1.4^{4}$ with parameters as defaults.

\section{Mobilization Assays of SGI1}

Many SGI1 variants can form the circular extrachromosomal forms that are conjugally mobilized in trans to other bacteria with the help of the conjugative IncA/C plasmid (Hall, 2010). The circular extrachromosomal forms of SGI1 variants were detected through two rounds of PCR amplification using primers listed in Supplementary Table S2. Mobilization assays were carried out as previously described (Siebor et al., 2016), using E. coli C600 harboring an IncC plasmid pR55 as recipient strain. Transconjugants were selected on Shigella and Salmonella agar plates containing $300 \mathrm{mg} / \mathrm{L}$ rifampicin and trimethoprim $(30 \mathrm{mg} / \mathrm{L}) /$ streptomycin $(30 \mathrm{mg} / \mathrm{L})$. The transfer frequency of SGI1was determined by dividing the number of E. coli SGI1 transconjugants by the number of $P$. mirabilis or $S$. enterica donor cells (Douard et al., 2010). The transconjugants were further

\footnotetext{
${ }^{1}$ https://cge.cbs.dtu.dk/services/MLST/

${ }^{2}$ https://cge.cbs.dtu.dk/services/ResFinder/

${ }^{3}$ http://blast.ncbi.nlm.nih.gov/Blast.cgi

${ }^{4}$ https://cge.cbs.dtu.dk/services/CSIPhylogeny/
} 
TABLE 1 | SGl1-containing S. enterica and P. mirabilis isolates characterized in this study.

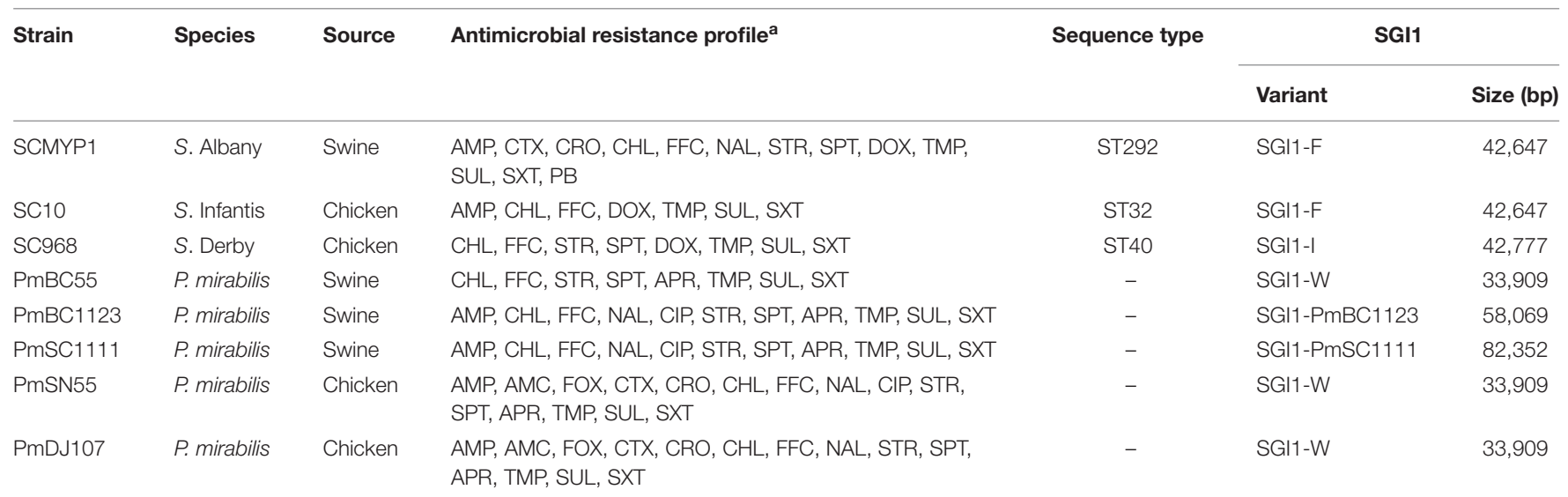

aproteus mirabilis is intrinsically resistant to doxycycline and polymyxin $B$.

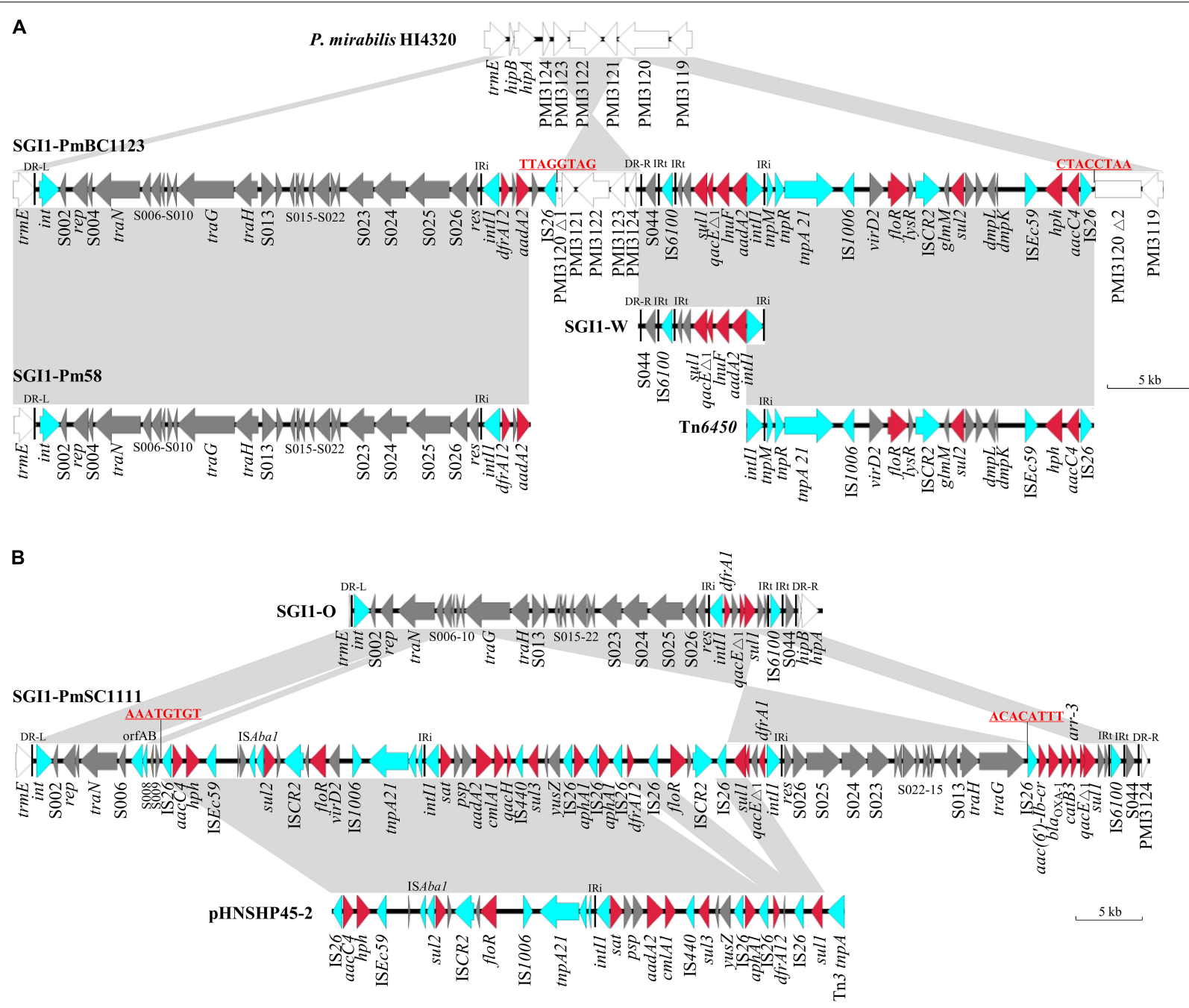

FIGURE 1 | Genetic structures of SGl1-PmBC1123 (A) and SGl1-PmSC1111 (B). Structures are drawn to scale from GenBank accession numbers MH998664 (SGl1-PmBC1123), MH998665 (SGl1-PmSC1111), AM942759 (P. mirabilis HI4320), KP662516 (SGl1-Pm58), MF805806 (Tn6450), KJ186151 (SGl1-W), KJ186150 (SGI1-O) and KU341381 (pHNSHP45-2). Genes and ORFs are shown as arrows, and their orientations of transcription are indicated by the arrowheads. Shared regions with above $99 \%$ identity are indicated by shading. Resistance genes are in red and transposase or integrase genes are in blue. DR-L and DR-R represent the 18-bp direct repeats at the ends of SGI1. IRi and IRt represent the inverted repeats defining the left and right ends of class 1 integron, respectively. 
examined for the presence of SGI1 integrase gene and the location of SGI1 in E. coli with primers listed in Supplementary Table S2.

\section{Nucleotide Sequence Accession Numbers}

The complete nucleotide sequences of two novel SGI1 variants and the genomes of $P$. mirabilis strains PmSC1111 and PmBC1123 were submitted to GenBank and assigned accession numbers MH998664 (SGI1-PmBC1123), MH998665 (SGI1-PmSC1111), CP034091 (PmBC1123) and CP034090 (PmSC1111), respectively. The Whole Genome Shotgun projects have been deposited at DDBJ/ENA/GenBank under the accession RQSE00000000 (SCMYP1), RQJQ00000000 (SC10), RQSD00000000 (SC968), RQSF00000000 (PmBC55), RQSG00000000 (PmSN55) and RQSH00000000 (PmDJ107).

\section{RESULTS AND DISCUSSION}

\section{Prevalence of SGI/PGI in S. enterica and P. mirabilis}

Detection and sequence analysis of SGI/PGI/AGI/GIPmi1 integrase gene showed that $3 \mathrm{~S}$. enterica and 5 P. mirabilis strains were positive for SGI1. Antimicrobial susceptibility testing indicated those 8 strains displayed different MDR profiles (Table 1) ( $P$. mirabilis is intrinsically resistant to doxycycline and polymyxin B). S. Albany strain SCMYP1 of swine origin exhibited resistance to the third generation cephalosporins and polymyxin B. Two $P$. mirabilis strains (PmSN55 and PmDJ107) of chicken origin were resistance to amoxicillin-clavulanic acid, cefoxitin and the third generation cephalosporins. Three SGI1-containg S. enterica strains belonged to different STs (Table 1). A total of 28,848 SNPs were called and phylogenetic analysis showed the five P. mirabilis strains were not clonally related.

\section{Characterization of SGI1 Variants}

Five different SGI1 variants were identified in the eight strains through whole genome sequencing and PCR linkage. S. Albany strain SCMYP1 (ST292) and S. Infantis strain SC10 (ST32) harbors SGI1 variant SGI1-F that carries $d f r A 1-$ orfC and bla $a_{\mathrm{CARB}-2}$ (ampicillin) gene cassettes, as well as resistance genes sull (sulfonamides), tet $A(G)$ (tetracycline) and floR (chloramphenicol and florfenicol). S. Derby strain SC968

TABLE 2 | Conjugative transfer frequency of S. enterica or P. mirabilis SGI1s.

\begin{tabular}{llll}
\hline Donor strain & SGl1 variant & Conjugative plasmid & $\begin{array}{c}\text { SGl1 transfer } \\
\text { frequency }\end{array}$ \\
\hline SCMYP1 & SGl1-F & IncC plasmid pR55 & $1.7 \times 10^{-6}$ \\
SC10 & SGl1-F & IncC plasmid pR55 & $1.9 \times 10^{-6}$ \\
SC968 & SGl1-I & IncC plasmid pR55 & $2.3 \times 10^{-7}$ \\
PmBC55 & SGl1-W & IncC plasmid pR55 & $4.3 \times 10^{-6}$ \\
PmSN55 & SGl1-W & IncC plasmid pR55 & $3.7 \times 10^{-6}$ \\
PmDJ107 & SGl1-W & IncC plasmid pR55 & $3.4 \times 10^{-6}$
\end{tabular}

(ST40) harbors SGI1 variant SGI1-I that carries gene cassettes aadA2 (streptomycin and spectinomycin) and dfrA1-orfC. Three P. mirabilis strains harbor SGI1 variant SGI1-W carrying aadA2$\ln u F$ gene cassettes. SGI1-W was firstly detected in P. mirabilis of poultry origin in China (Lei et al., 2014), and then found in P. mirabilis and P. stuartii in Egypt (Soliman et al., 2017, 2019), suggesting this variant of SGI1 had the potential to spread among enterobacterial strains worldwide. It is interesting that two novel SGI1 variants, SGI1-PmBC1123 and SGI1-PmSC1111 (Figure 1), are characterized in this study for the first time.

SGI1-PmBC1123 is 58,069 bp in size (corresponding to bases 1,348-32,501 and 37,667-64,581 in accession no. MH998664) that carries gene cassettes $d f$ rA12-orfF-aadA2 and aadA2-lnuF found in SGI1-Z (Qin et al., 2015) and SGI1-W (Lei et al., 2014), respectively. The MDR region of SGI1-PmBC1123 is divided into two parts separated by a chromosomal DNA fragment of 5,148 bp (truncated PMI3120 and PMI3121-PMI3124). We hypothesize that an initial single IS26 transposition event occurred in the chromosome in locus PMI3120 and has subsequently generated an inversion between two IS26 elements in opposite orientation, resulting in the right end of SGI1-PmBC1123 and this 5,148 bp chromosomal DNA fragment being in inverse orientation. The similar phenomenon is also found in PGI1 (Siebor and Neuwirth, 2014). The $20.74 \mathrm{~kb}$ region in SGI1-PmBC1123 (corresponding to bases 43,839-64,581 in accession no. MH998664) that carries floR, sul2 (sulfonamides), $h p h$ (hygromycin) and aacC4 (apramycin) shows $99.9 \%$ nucleotide identity to the corresponding region of Tn6450 (Chen et al., 2018), indicating they might have a common origin.

SGI1-PmSC1111 is 82,352 bp in size (corresponding to bases 1,348-83,699 in accession no. MH998665). A 1,258 bp insertion sequence, encoding transposase OrfAB subunits A and $\mathrm{B}$, was inserted into the backbone gene $\mathrm{S} 007$ and flanked by 3-bp target site duplication (AAG). This IS element shows 98.6\% nucleotide identity to ISalg firstly described in Vibrio cholerae serogroup O103 (Sozhamannan et al., 1999), and 86\% nucleotide identity to ISVch4 in some SGI1 variants like SGI1K (Doublet et al., 2008). An IS26-mediated recombination event occurred in S010, which caused the middle region of SGI1PmSC1111 (corresponding to bases 10,880-75,577 in accession no. MH998665) being in inverse orientation and flanked by 8 bp inverted repeats (AAATGTGT) (Figure 1B). The downstream region of the 3'-partial of S010 (corresponding to bases 10,88054,663 in accession no. MH998665) shows $99.9 \%$ nucleotide identity to the corresponding region of pHNSHP45-2 (accession no. KU341381) with the addition of two regions, aphA1-IS26 and floR-ISCR2-IS26. It harbors an atypical class 1 integron ( $5^{\prime} \mathrm{CS}$-satpsp-aadA2-cmlA1-aadA1-qacH-IS440-sul3) belonging to sul3type III (Antunes et al., 2007). The class 1 integron adjacent to the res gene (S027) carries gene cassettes dfrA1-orfC. This region contains five copies of IS26 in the same orientation. However, the 8-bp target site duplications could not be observed around these IS26, suggesting this complicated MDR region might be formed via the incorporation of several IS26-mediated translocatable units successively (Harmer and Hall, 2015, 2016). The right end of SGI1-PmSC1111 harbors four gene cassettes, aac $\left(6^{\prime}\right)-\mathrm{Ib}-\mathrm{cr}$ (fluoroquinolones and aminoglycosides), bla $a_{\mathrm{OXA}-1}$ (ampicillin), 
TABLE 3 | Acquired antimicrobial resistance genes in SGl1-containing S. enterica and P. mirabilis isolates.

\begin{tabular}{|c|c|c|c|c|c|c|c|c|c|c|}
\hline Strain & Aminoglycoside $^{a}$ & $\beta$-lactam & Colistin & Fluoroquinolone & $\begin{array}{l}\text { MLS - Macrolide, } \\
\text { Lincosamide and } \\
\text { Streptogramin B }\end{array}$ & Phenicol & Rifampicin & Sulfonamide & Tetracycline & Trimethoprim \\
\hline SCMYP1 & & $\begin{array}{l}\text { blacARB-2 } \\
\text { blactX-M-55 }\end{array}$ & $m c r-1$ & & & floR & & sul1 & $\operatorname{tet} A(\mathrm{G})$ & dfra1 \\
\hline SC10 & & $b_{\text {CARB-2 }}$ & & & & floR & & sul1 & $\operatorname{tet} A(\mathrm{G})$ & dfrA1 \\
\hline SC968 & aadA2 & & & & & floR & & sul1 & $\operatorname{tet} A(\mathrm{G})$ & dfrA1 \\
\hline PmBC55 & $\begin{array}{l}\text { strB } \\
\text { strA } \\
\text { hph } \\
\text { aacC4 } \\
\text { aadA1 } \\
\text { aadA2 }\end{array}$ & & & & $\ln u(F)$ & $\begin{array}{l}\text { floR } \\
\text { catB2 }\end{array}$ & & $\begin{array}{l}\text { sul1 } \\
\text { sul2 }\end{array}$ & $\operatorname{tet} A(\mathrm{~A})$ & $\begin{array}{l}\text { dfrA12 } \\
\text { dfrA1 }\end{array}$ \\
\hline PmBC1123 & $\begin{array}{l}\text { aphA1 } \\
\text { strA } \\
\text { strB } \\
\text { aacC4 } \\
\text { hph } \\
\text { aadA2 }\end{array}$ & blaoXA-1 & & $\operatorname{aac}\left(6^{\prime}\right)-1 b-c r$ & $\begin{array}{l}\operatorname{ere}(A) \\
\ln u(F)\end{array}$ & $\begin{array}{l}\text { catB4 } \\
\text { floR }\end{array}$ & arr-3 & $\begin{array}{l}\text { sul1 } \\
\text { sul2 }\end{array}$ & $\operatorname{tet} A(\mathrm{C})$ & $\begin{array}{l}\text { dfrA12 } \\
\text { dfrA32 }\end{array}$ \\
\hline PmSC1111 & $\begin{array}{l}\text { hph } \\
\text { aacC4 } \\
\text { aphA1 } \\
\text { strA } \\
\text { strB } \\
\text { aadA1 } \\
\text { aadA2 }\end{array}$ & blaoxA-1 & & $a a c\left(6^{\prime}\right)-l b-c r$ & $\begin{array}{l}\operatorname{ere}(A) \\
c f r \\
m p h(E) \\
m s r(E)\end{array}$ & $\begin{array}{l}\text { floR } \\
\text { cmlA1 } \\
\text { catB3 }\end{array}$ & arr-3 & $\begin{array}{l}\text { sul1 } \\
\text { sul2 } \\
\text { sul3 }\end{array}$ & $\begin{array}{l}\operatorname{tet} A(\mathrm{C}) \\
\operatorname{tet} A(\mathrm{D})\end{array}$ & $\begin{array}{l}\text { dfrA12 } \\
\text { dfrA32 } \\
\text { dfrA1 }\end{array}$ \\
\hline PmSN55 & $\begin{array}{l}\text { hph } \\
\text { aacC4 } \\
\text { aphA1 } \\
\text { aadA2 } \\
\text { aadA14 }\end{array}$ & bla $\mathrm{CMY}_{-2}$ & & & $\ln u(F)$ & floR & & $\begin{array}{l}\text { sul1 } \\
\text { sul2 }\end{array}$ & & dfrA12 \\
\hline PmDJ107 & $\begin{array}{l}\text { hph } \\
\text { aacC4 } \\
\text { aphA1 } \\
\text { aadA1 } \\
\text { aadA2 }\end{array}$ & bla $\mathrm{CMY}-2$ & & & $\ln u(F)$ & floR & & $\begin{array}{l}\text { sul1 } \\
\text { sul2 }\end{array}$ & & $\begin{array}{l}\text { dfrA12 } \\
\text { dfrA1 }\end{array}$ \\
\hline
\end{tabular}

${ }^{a}$ Antimicrobial resistance genes carried by SG/1 are indicated in bold. tetA(J) is inherent in the chromosome. ${ }^{b}$ Acquired antimicrobial resistance genes were identified by ResFinder 3.1 (https://cge.cbs.dtu.dk/services/ ResFinder/). 
catB3 (chloramphenicol) and arr-3 (rifampicin) never reported to date in SGI1.

\section{Mobilization of SGl1}

A free circle can be formed after excision of SGI1 from the chromosome (Doublet et al., 2005). SGI1 appeared to be nonself-transmissible, but it could potentially be integrated into the chromosome of another bacterial species by the help of IncA/C plasmid (Douard et al., 2010; Siebor et al., 2016). In the recipient strain, the circular form of SGI1 integrates in a specific site at the $3^{\prime}$ end of the chromosomal trmE gene (Doublet et al., 2005).

The circular forms of SGI1 in all strains except for PmBC1123 and PmSC1111 were detected by two rounds of PCR amplification. We did not detect the circular form of SGI1-PmBC1123 because of the inversion of the right direct repeat in SGI1-PmBC1123. The circular form of SGI1PmSC1111 could not be detected through three independent experiments. Mobilization assays showed that the three known SGI1 variants (SGI1-F, SGI1-I and SGI1-W) in $S$. enterica or $P$. mirabilis could be conjugally mobilized to E. coli and was incorporated into the $3^{\prime}$-end of trmE. The conjugative transfer of them were detected at frequencies between $10^{-6}$ and $10^{-7}$, suggesting that these SGI1s can be transferred between bacterial species (Table 2). However, the mobilization of SGI1-PmBC1123 and SGI1-PmSC1111 failed despite three independent attempts. The results indicate that the sequence inversion of partial SGI1 backbone may result in the loss of mobility of SGI1. We supposed that the inversion of the right direct repeat in SGI1-PmBC1123 may lead to losing the capability to form a circular form and then mobility. Nevertheless, in SGI1-PmSC1111, IS26mediated rearrangements resulted in inversions of the backbone was supposed to affect the expression of some genes related to mobilization. The mechanism needs further study to clarify.

\section{Other Resistance Genes That Were Not Associated With SGI1}

The acquired antimicrobial resistance genes in SGI1-containing $S$. enterica and $P$. mirabilis isolates are listed in Table 3 . S. Albany strain SCMYP1 harbors bla $a_{\mathrm{CTX}-\mathrm{M}-55}$ and $m c r-1$, explaining the resistance to third generation cephalosporins and polymyxin $\mathrm{B}$, respectively. $P$. mirabilis strains PmSN55 and PmDJ107 harbors AmpC cephalosporinase gene bla $\mathrm{CMY}-2$ carried by an 11.7-kb contig that is identical to the corresponding region of SXT/R391 ICE ICEPmiJpn1 (Harada et al., 2010; Lei et al., 2016), indicating the bla $a_{\mathrm{CMY}-2}$ gene in those two strains might be carried by ICEPmiJpn1. It is notable that strain PmSC1111 harbors the multiresistance gene $c f r$ that is also carried by SXT/R391 ICE (accession no. CP034090). Very recently we reported a novel SXT/R391 ICE that carried $c f r$, $b l a_{\mathrm{CTX}-\mathrm{M}-65}$, fos $A 3$, and $a a c\left(6^{\prime}\right)-I b-c r$ in P. mirabilis and could be transferred to E. coli (Lei et al., 2018a). Taken together, SXT/R391 ICE could mediate the dissemination of clinically important resistance genes in $P$. mirabilis, which needs to draw more attention.

\section{CONCLUSION}

In this study, we characterized SGI1 in S. enterica and P. mirabilis of food-producing animal origin in Sichuan Province and described two novel SGI1 variants, SGI1-PmBC1123 and SGI1-PmSC1111. The sul3-type III class 1 integron (5'CS-sat-psp-aadA2-cmlA1-aadA1-qacH-IS440-sul3) and gene cassettes $a a c\left(6^{\prime}\right)-I b-c r-b l a_{\mathrm{OXA}-1}-c a t B 3-a r r-3$ are reported in SGI1 for the first time. Our study highlights that IS26-mediated rearrangements promote the diversity of SGI1.

\section{DATA AVAILABILITY STATEMENT}

The datasets generated for this study can be found in the GeneBank, MH998664, MH998665, CP034091 and CP034090.

\section{ETHICS STATEMENT}

This study was carried out in accordance with the recommendation of ethical guidelines of Sichuan University. The protocol was approved by the Sichuan University Animal Ethics Committee. Individual informed consent for the use of samples was obtained from all the animal owners.

\section{AUTHOR CONTRIBUTIONS}

$\mathrm{X}-\mathrm{CW}, \mathrm{C}-\mathrm{WL}, \mathrm{Z}-\mathrm{ZK}$, and YZ performed the experiments. C-WL analyzed the data and conceived of the study. X-CW, C-WL, and Z-ZK wrote the manuscript. All authors contributed to manuscript revision and approved the final manuscript.

\section{FUNDING}

This work was supported by the General Program of National Natural Science Foundation of China (Grant No. 31572547 and 31830098), the International Collaborative Program of Sichuan Province (Grant No. 2018HH0027), the Scientific Research Foundation of Sichuan University (Grant No. 2017SCU12006), the China Postdoctoral Science Foundation (Grant No. 2017M623036), and the Fundamental Research Funds for the Central Universities (Grant No. SCU2019D013).

\section{ACKNOWLEDGMENTS}

The authors wish to thank Professor Yang Wang from China Agricultural University for generously donating pR55-containing E. coli C600 strain.

\section{SUPPLEMENTARY MATERIAL}

The Supplementary Material for this article can be found online at: https://www.frontiersin.org/articles/10.3389/fmicb.2019. 02245/full\#supplementary-material 


\section{REFERENCES}

Ahmed, A. M., Hussein, A. I., and Shimamoto, T. (2007). Proteus mirabilis clinical isolate harbouring a new variant of Salmonella genomic island 1 containing the multiple antibiotic resistance region. J. Antimicrob. Chemother. 59, 184-190. doi: 10.1093/jac/dkl471

Antunes, P., Machado, J., and Peixe, L. (2007). Dissemination of sul3-containing elements linked to class 1 integrons with an unusual 3' conserved sequence region among Salmonella isolates. Antimicrob. Agents Chemother. 51, 15451548. doi: 10.1128/aac.01275-1276

Bellanger, X., Payot, S., Leblond-Bourget, N., and Guedon, G. (2014). Conjugative and mobilizable genomic islands in bacteria: evolution and diversity. FEMS Microbiol. Rev. 38, 720-760. doi: 10.1111/1574-6976.12058

Bi, S., Yan, H., Chen, M., Zhang, Z., Shi, L., and Wang, H. (2011). New variant Salmonella genomic island 1-U in Proteus mirabilis clinical and food isolates from South China. J. Antimicrob. Chemother. 66, 1178-1179. doi: 10.1093/jac/ dkr030

Bie, L., Fang, M., Li, Z., Wang, M., and Xu, H. (2018). Identification and characterization of new resistance-conferring SGI1s (Salmonella genomic Island 1) in Proteus mirabilis. Front. Microbiol. 9:3172. doi: 10.3389/fmicb.2018. 03172

Boyd, D., Peters, G. A., Cloeckaert, A., Boumedine, K. S., Chaslus-Dancla, E., Imberechts, H., et al. (2001). Complete nucleotide sequence of a 43-kilobase genomic island associated with the multidrug resistance region of Salmonella enterica serovar typhimurium DT104 and its identification in phage type DT120 and serovar Agona. J. Bacteriol. 183, 5725-5732. doi: 10.1128/jb.183.19.57255732.2001

Carraro, N., Matteau, D., Luo, P., Rodrigue, S., and Burrus, V. (2014). The master activator of IncA/C conjugative plasmids stimulates genomic islands and multidrug resistance dissemination. PLoS Genet. 10:e1004714. doi: 10.1371/ journal.pgen.1004714

Chen, Y. P., Lei, C. W., Kong, L. H., Zeng, J. X., Zhang, X. Z., Liu, B. H., et al. (2018). Tn6450, a novel multidrug resistance transposon characterized in a Proteus mirabilis isolate from chicken in China. Antimicrob. Agents Chemother. 62:e2192-17. doi: 10.1128/aac.02192-2117

Cummins, M. L., Roy Chowdhury, P., Marenda, M. S., Browning, G. F., and Djordjevic, S. P. (2019). Salmonella genomic island 1B variant found in a sequence type 117 avian pathogenic Escherichia coli isolate. mSphere 4:e169-19. doi: $10.1128 / \mathrm{mSphere}$.00169-119

de Curraize, C., Neuwirth, C., Bador, J., Chapuis, A., Amoureux, L., and Siebor, E. (2018). Two new Salmonella genomic islands 1 from Proteus mirabilis and description of blaCTX-M-15 on a variant (SGI1-K7). J. Antimicrob. Chemother. 73, 1804-1807. doi: 10.1093/jac/dky108

Douard, G., Praud, K., Cloeckaert, A., and Doublet, B. (2010). The Salmonella genomic island 1 is specifically mobilized in trans by the IncA/C multidrug resistance plasmid family. PLoS One 5:e15302. doi: 10.1371/journal.pone. 0015302

Doublet, B., Boyd, D., Mulvey, M. R., and Cloeckaert, A. (2005). The Salmonella genomic island 1 is an integrative mobilizable element. Mol. Microbiol. 55, 1911-1924. doi: 10.1111/j.1365-2958.2005.04 520.x

Doublet, B., Praud, K., Bertrand, S., Collard, J. M., Weill, F. X., and Cloeckaert, A. (2008). Novel insertion sequence- and transposon-mediated genetic rearrangements in genomic island SGI1 of Salmonella enterica serovar kentucky. Antimicrob. Agents Chemother. 52, 3745-3754. doi: 10.1128/aac. 00525-528

Girlich, D., Dortet, L., Poirel, L., and Nordmann, P. (2015). Integration of the blaNDM-1 carbapenemase gene into proteus genomic island 1 (PGI1-PmPEL) in a Proteus mirabilis clinical isolate. J. Antimicrob. Chemother. 70, 98-102. doi: $10.1093 / \mathrm{jac} / \mathrm{dku} 371$

Hall, R. M. (2010). Salmonella genomic islands and antibiotic resistance in Salmonella enterica. Future Microbiol. 5, 1525-1538. doi: 10.2217/fmb. 10.122

Hamidian, M., Holt, K. E., and Hall, R. M. (2015). Genomic resistance island AGI1 carrying a complex class 1 integron in a multiply antibiotic-resistant ST25 Acinetobacter baumannii isolate. J. Antimicrob. Chemother. 70, 2519-2523. doi: $10.1093 / \mathrm{jac} / \mathrm{dkv} 137$
Harada, S., Ishii, Y., Saga, T., Tateda, K., and Yamaguchi, K. (2010). Chromosomally encoded blaCMY-2 located on a novel SXT/R391-related integrating conjugative element in a Proteus mirabilis clinical isolate. Antimicrob. Agents Chemother. 54, 3545-3550. doi: 10.1128/aac.00111-110

Harmer, C. J., and Hall, R. M. (2015). ). IS26-mediated precise excision of the IS26-aphA1a translocatable unit. mBio 6:e1866-15. doi: 10.1128/mBio.018661815

Harmer, C. J., and Hall, R. M. (2016). IS26-mediated formation of transposons carrying antibiotic resistance genes. $m$ Sphere 1:e38-16. doi: 10.1128/mSphere. 00038- 16

Kiss, J., Papp, P. P., Szabo, M., Farkas, T., Muranyi, G., Szakallas, E., et al. (2015). The master regulator of IncA/C plasmids is recognized by the Salmonella genomic island SGI1 as a signal for excision and conjugal transfer. Nucleic Acids Res. 43, 8735-8745. doi: 10.1093/nar/gkv758

Kiss, J., Szabo, M., Hegyi, A., Douard, G., Praud, K., Nagy, I., et al. (2019). Identification and characterization of oriT and Two mobilization genes required for conjugative transfer of Salmonella genomic island 1. Front. Microbiol. 10:457. doi: 10.3389/fmicb.2019.00457

Lei, C. W., Chen, Y. P., Kang, Z. Z., Kong, L. H., and Wang, H. N. (2018a). Characterization of a Novel SXT/R391 Integrative and conjugative element carrying cfr, blaCTX-M-65, fosA3, and aac $\left(6^{\prime}\right)-\mathrm{Ib}-\mathrm{cr}$ in Proteus mirabilis. Antimicrob. Agents Chemother. 62:e849-18. doi: 10.1128/aac.00849-818

Lei, C. W., Chen, Y. P., Kong, L. H., Zeng, J. X., Wang, Y. X., Zhang, A. Y., et al. (2018b). PGI2 Is a Novel SGI1-Relative multidrug-resistant genomic island characterized in Proteus mirabilis. Antimicrob. Agents Chemother. 62:e19-18. doi: 10.1128/aac.00019-18

Lei, C. W., Zhang, A. Y., Liu, B. H., Wang, H. N., Guan, Z. B., Xu, C. W., et al. (2014). Molecular characteristics of Salmonella genomic island 1 in Proteus mirabilis isolates from poultry farms in China. Antimicrob. Agents Chemother. 58, 7570-7572. doi: 10.1128/aac.03992-3914

Lei, C. W., Zhang, A. Y., Liu, B. H., Wang, H. N., Yang, L. Q., Guan, Z. B., et al. (2015). Two novel Salmonella genomic island 1 variants in Proteus mirabilis isolates from swine farms in China. Antimicrob. Agents Chemother. 59, 4336-4338. doi: 10.1128/aac.00120-115

Lei, C. W., Zhang, A. Y., Wang, H. N., Liu, B. H., Yang, L. Q., and Yang, Y. Q. (2016). Characterization of SXT/R391 integrative and conjugative elements in Proteus mirabilis isolates from food-producing animals in China. Antimicrob. Agents Chemother. 60, 1935-1938. doi: 10.1128/aac.02852-2815

Levings, R. S., Djordjevic, S. P., and Hall, R. M. (2008). SGI2, a relative of Salmonella genomic island SGI1 with an independent origin. Antimicrob. Agents Chemother. 52, 2529-2537. doi: 10.1128/aac.00189-188

Levings, R. S., Lightfoot, D., Partridge, S. R., Hall, R. M., and Djordjevic, S. P. (2005). The genomic island SGI1, containing the multiple antibiotic resistance region of Salmonella enterica serovar typhimurium DT104 or variants of it, is widely distributed in other S. enterica serovars. J. Bacteriol. 187, 4401-4409. doi: 10.1128/jb.187.13.4401-4409.2005

Partridge, S. R., Kwong, S. M., Firth, N., and Jensen, S. O. (2018). Mobile genetic elements associated with antimicrobial resistance. Clin. Microbiol. Rev. 31:e8817. doi: $10.1128 / \mathrm{cmr} .00088-17$

Qin, S., Qi, H., Zhang, Q., Zhao, D., Liu, Z. Z., Tian, H., et al. (2015). Emergence of extensively drug-resistant Proteus mirabilis harboring a conjugative NDM-1 plasmid and a novel Salmonella genomic island 1 variant, SGI1-Z. Antimicrob. Agents Chemother. 59, 6601-6604. doi: 10.1128/aac.002 92-215

Schultz, E., Barraud, O., Madec, J. Y., Haenni, M., Cloeckaert, A., Ploy, M. C., et al. (2017a). Multidrug resistance Salmonella genomic island 1 in a Morganella morganii subsp. morganii human clinical isolate from France. mSphere 2:e11817. doi: 10.1128/mSphere.00118-117

Schultz, E., Cloeckaert, A., Doublet, B., Madec, J. Y., and Haenni, M. (2017b). Detection of SGI1/PGI1 elements and resistance to extended-spectrum cephalosporins in proteae of animal origin in France. Front. Microbiol. 8:32. doi: $10.3389 /$ fmicb.2017.00032

Schultz, E., Haenni, M., Mereghetti, L., Siebor, E., Neuwirth, C., Madec, J. Y., et al. (2015). Survey of multidrug resistance integrative mobilizable elements SGI1 and PGI1 in Proteus mirabilis in humans and dogs in France, 2010-13. J. Antimicrob. Chemother. 70, 2543-2546. doi: 10.1093/jac/dk v154 
Siebor, E., de Curraize, C., Amoureux, L., and Neuwirth, C. (2016). Mobilization of the Salmonella genomic island SGI1 and the proteus genomic island PGI1 by the A/C2 plasmid carrying blaTEM-24 harboured by various clinical species of Enterobacteriaceae. J. Antimicrob. Chemother. 71, 2167-2170. doi: 10.1093/jac/ dkw151

Siebor, E., de Curraize, C., and Neuwirth, C. (2018). Genomic context of resistance genes within a French clinical MDR Proteus mirabilis: identification of the novel genomic resistance island GIPmil. J. Antimicrob. Chemother. 73, 1808-1811. doi: $10.1093 / \mathrm{jac} / \mathrm{dky} 126$

Siebor, E., de Curraize, C., and Neuwirth, C. (2019). Identification of AGI1-A, a variant of acinetobacter genomic island 1 (AGI1), in a French clinical isolate belonging to the Enterobacter cloacae complex. J. Antimicrob. Chemother. 74, 311-314. doi: 10.1093/jac/dky442

Siebor, E., and Neuwirth, C. (2011). The new variant of Salmonella genomic island 1 (SGI1-V) from a Proteus mirabilis French clinical isolate harbours blaVEB-6 and qnrA1 in the multiple antibiotic resistance region. J. Antimicrob. Chemother. 66, 2513-2520. doi: 10.1093/jac/dkr335

Siebor, E., and Neuwirth, C. (2013). Emergence of Salmonella genomic island 1 (SGI1) among Proteus mirabilis clinical isolates in Dijon. France. J. Antimicrob. Chemother. 68, 1750-1756. doi: 10.1093/jac/dkt100

Siebor, E., and Neuwirth, C. (2014). Proteus genomic island 1 (PGI1), a new resistance genomic island from two Proteus mirabilis French clinical isolates. J. Antimicrob. Chemother. 69, 3216-3220. doi: 10.1093/jac/dk u314
Soliman, A. M., Ahmed, A. M., Shimamoto, T., El-Domany, R. A., Nariya, H., and Shimamoto, T. (2017). First report in Africa of two clinical isolates of Proteus mirabilis carrying Salmonella genomic island (SGI1) variants. SGI1-PmABB and SGI1-W. Infect. Genet. Evol. 51, 132-137. doi: 10.1016/j.meegid.2017. 03.029

Soliman, A. M., Shimamoto, T., Nariya, H., and Shimamoto, T. (2019). Emergence of Salmonella genomic island 1 variant SGI1-W in a clinical Isolate of providencia stuartii from Egypt. Antimicrob. Agents Chemother. 63:e1793-18. doi: 10.1128/aac.01793-1718

Sozhamannan, S., Deng, Y. K., Li, M., Sulakvelidze, A., Kaper, J. B., Johnson, J. A., et al. (1999). Cloning and sequencing of the genes downstream of the wbf gene cluster of Vibrio cholerae serogroup O139 and analysis of the junction genes in other serogroups. Infect. Immun. 67, 5033-5040.

Conflict of Interest: The authors declare that the research was conducted in the absence of any commercial or financial relationships that could be construed as a potential conflict of interest.

Copyright (C) 2019 Wang, Lei, Kang, Zhang and Wang. This is an open-access article distributed under the terms of the Creative Commons Attribution License (CC BY). The use, distribution or reproduction in other forums is permitted, provided the original author(s) and the copyright owner(s) are credited and that the original publication in this journal is cited, in accordance with accepted academic practice. No use, distribution or reproduction is permitted which does not comply with these terms. 